\title{
Retraction
}

\section{Retraction: Zeng et al., Epigenetic Enhancement of BDNF Signaling Rescues Synaptic Plasticity in Aging}

The Journal of Neuroscience has received a report of an investigation by the University of California, Los Angeles that describes substantial data misrepresentation in the article "Epigenetic Enhancement of BDNF Signaling Rescues Synaptic Plasticity in Aging" by Yan Zeng, Miao Tan, Jun Kohyama, Marissa Sneddon, Joseph B. Watson, Yi E. Sun, and Cui-Wei Xie, which appeared on pages 17800-17810 of the December 7, 2011 issue. Because the results cannot be considered reliable, the editors of The Journal are retracting the paper.

DOI: 10.1523/JNEUROSCI.4832-13.2013 